\title{
(Sub)mm-Wavelength Observations of Pre-Planetary Nebulae and Young Planetary Nebulae
}

\author{
Carmen Sánchez Contreras \\ Centro de Astrobiología (CSIC-INTA), Camino Bajo del Castillo s/n, Urb. Villafranca del Castillo, \\ Villanueva de la Cañada, E-28691 Madrid, Spain; csanchez@cab.inta-csic.es
}

Received: 17 February 2020; Accepted: 28 February 2020; Published: 10 March 2020

\begin{abstract}
This is a non-comprehensive review of observations of pre-Planetary Nebulae ( $\mathrm{pPNe})$ and young Planetary Nebulae (yPNe) at (sub)mm-wavelengths, a valuable window for probing multi-phased gas and dust in these objects. This contribution focuses on observations of molecular lines (from carbon monoxide- $\mathrm{CO}$ - and other species), and briefly at the end, on hydrogen radio recombination lines from the emerging $\mathrm{HII}$ regions at the center of yPNe. The main goal of this contribution is to show the potential of (sub)mm-wavelength observations of pPNe/yPNe to help the community to devise and develop new observational projects that will bring us closer to a better understanding of these latest stages of the evolution of low-to-intermediate $\left(\sim 0.8-8 M_{\odot}\right)$ mass stars.
\end{abstract}

Keywords: AGB and post-AGB stars; circumstellar matter; winds and outflows; mass-loss; jets

\section{Introduction}

In this contribution, I will show a few examples of observations of pre-Planetary Nebulae (pPNe) and young $\mathrm{PNe}(\mathrm{yPNe})$ at (sub)mm-wavelengths, a spectral domain that is particularly useful for probing the gas (at multiple phases) and dust present in these objects. For general reviews of the evolution from the asymptotic giant branch (AGB) to the PN phase and the major open questions in the field, the reader is referred to, e.g., Balick \& Frank [1], Kwok [2], van Winckel [3].

I will overview molecular line observations (Section 2) and will describe our current view of the nebular structure and dynamics based on carbon monoxide (CO) emission observations (Section 2.1) in two major classes of post-AGB objects: the so-called normal or standard pPNe (with massive, fast outflows) and disk-prominent post-AGB objects (with rotating circumbinary disks and tenuous outflows). I will describe with some detail recent interferometric, high-angular resolution observations of pPNe that reveal very complex nebular substructures indicative of an equally complex formation and evolution history. I will continue with a brief report on observations of molecular species other than $\mathrm{CO}$ detected in pPNe/yPNe (Section 2.2). In Section 3, I will describe recent observations of radio recombination lines at $\mathrm{mm}$-wavelengths showing that these are very useful probes of the central regions of yPNe, at scales of a few hundred AU. In Section 4, some final remarks are presented.

Disclaimer: this is not a comprehensive review and is probably biased by my personal limited view / knowledge of the field. Observations of molecular masers or polarization studies of pPNe are not covered in this contribution (see relevant contributions in this volume presented by other authors).

\section{Molecular Gas Component}

It is now well established that most of the nebular material in pPNe and yPNe is in the form of relatively cold (few tens of $\mathrm{K}$ ) molecular gas and that the low- $J$ transitions of $\mathrm{CO}$ at (sub)mm-wavelengths are excellent tracers of this component (for a review on this topic, see, e.g., [4] and references therein). Since the pioneering works in the 80 s-90s 
by Bachiller et al. [5], Bujarrabal et al. [6], Cernicharo et al. [7], Knapp et al. [8], Likkel et al. [9], Volk et al. [10], Huggins et al. [11], etc., CO emission observations have been widely used to study the nebular structure and dynamics of $\mathrm{pPNe} / \mathrm{yPNe}$. For many objects, in addition to $\mathrm{CO}$, a variety of molecular species have been detected, denoting a considerable chemical activity in these environments. A succinct overview of these topics is offered in the next subsections.

\subsection{Nebular Structure and Dynamics}

The first single-dish observations of $\mathrm{CO}$ line emission from $\mathrm{pPNe} / \mathrm{yPNe}$ revealed some important differences with respect to those from the envelopes around their progenitor AGB stars. The CO profiles of most $\mathrm{pPNe} / \mathrm{yPNe}$, which are then referred to as normal or standard, exhibit narrow (FWHM 15-30 $\mathrm{km} \mathrm{s}^{-1}$ ) intense line cores, which are identified with the remnant of the slow AGB wind. In contrast to AGB stars, however, $\mathrm{pPNe} / \mathrm{yPNe}$ show $\mathrm{CO}$ line cores bounded with weak but broad emission wings (with full widths of up to $\approx 100 \mathrm{~km} \mathrm{~s}^{-1}$ in some cases) indicative of the presence of fast outflows beyond the AGB stage. The $\mathrm{CO}$ emission profiles of $\mathrm{pPNe} / \mathrm{yPNe}$ are found to be in general somewhat structured (see e.g., [12]), suggesting nebular morphokinematics more complex than the slow, roughly round envelopes of AGB stars. Most pPNe are unresolved with single-dish telescopes, and therefore, interferometric mapping is required for a proper study of their nebular properties.

To date, about $30-40$ pPNe have been mapped with moderate $\left(\leqslant 5-10^{\prime \prime}\right)$ angular resolution; however, only a fraction of these $(\sim 40 \%)$ are mapped with enough angular resolution $\left(\sim 1^{\prime \prime}\right)$ to study in some detail their nebular structure and to isolate different components. Some examples of interferometric observations can be found in Castro-Carrizo et al. [13], Cox et al. [14], Fong et al. [15], Huggins et al. [16], Neri et al. [17], Sánchez Contreras et al. [18], Sánchez Contreras \& Sahai [19], and Tafoya et al. [20]; a few more are described in some detail below.

Interferometric maps show that the broad $\mathrm{CO}$ emission wings of $\mathrm{pPNe} / \mathrm{yPNe}$ typically arise in elongated structures or lobes, which are commonly interpreted as part of the material originally in the AGB envelope that has been sculpted and accelerated by underlying fast and collimated (jet-like) post-AGB ejections. The slow component that produces the narrow core of the $\mathrm{CO}$ emission normally arises in the equatorial regions of the nebulae where the bulk of the gas is radially expanding and probably represents the circumstellar material that has not been largely affected by the interaction with fast winds. A few examples of $\mathrm{CO}$ outflows from standard $\mathrm{pPNe}$ are given in the following.

The pPN M1-92 [21] is one of the best characterized pPNe and is considered by many the prototype of the so-called hourglass morphology (i.e., fast shell-like lobes and a slowly expanding equatorial waist/torus). Other examples of a similar morphology are M 2-56 [22], IRAS 15103-5754 [23], the Boomerang Nebula [24], and M 2-9 [25]. In the case of M2-9, the CO is fully photodissociated by the interstellar UV radiation field in the lobes, and it only survives in the densest equatorial regions. It is interesting to note the presence of not only one but two nested ring-like equatorial components in M2-9. These two rings are off-centered and have different systemic velocities, consistent with the rings being the results from two brief $(\sim 40 \mathrm{yr})$ mass ejection events that took place at different times during the orbital motion of the binary at the center [25].

The kinematics of the fast bipolar outflows of pPNe are well characterized for a number of cases. The pPN CRL 618 [26] is one of these, tidily showing that the expansion velocity increases linearly with the distance to the center along its bipolar outflow $\left(V_{\exp } \propto r\right)$. The kinematics seem to be common to most $\mathrm{pPNe} / \mathrm{yPNe}$ and are consistent with the jet+'AGB CSE' interaction scenario proposed to explain the bipolar shapes of pPNe and PNe (e.g., [1], and references therein). For most pPNe, the velocity gradients measured imply quite short kinematic ages of the order of $t_{\mathrm{kin}} \sim 1000 \mathrm{yr}$ or less (e.g., as short as $t_{\text {kin }} \sim 30-80 \mathrm{yr}$ in CRL 618), and possibly shorter ejections times of only a fraction of $t_{\text {kin }}$.

Another important characteristic of the majority $(\sim 80 \%)$ of standard pPNe is that the linear momentum carried by their fast $\left(\sim 100 \mathrm{~km} \mathrm{~s}^{-1}\right)$ outflows is larger than that available by radiation pressure on dust, which is the main source of momentum in AGB winds [12]. This means that the yet unknown mass-loss mechanism during the post-AGB phase is not the same as in the earlier 
AGB stage. The linear momentum excess problem is very pronounced in some objects; for example, IRAS 19374+2356, having the largest linear momentum measured to date $\left(\gtrsim 45 \mathrm{M}_{\odot} \mathrm{km} \mathrm{s}^{-1}\right)$ and having about $80 \%$ of the total nebular mass accelerated to velocities of $\gtrsim 100 \mathrm{~km} \mathrm{~s}^{-1}$ [27]. However, it is important to note that not all pPNe exhibit nebular properties as extreme as IRAS 19374+2356. Using CO emission (interferometric and single-dish) data, Sánchez Contreras \& Sahai [19] found that the fraction of gas accelerated to large velocities is below $\sim 20 \%$ in most pPNe. Moreover, although there is a number of pPNe with outflow speeds exceeding $\sim 100 \mathrm{~km} \mathrm{~s}^{-1}$, the average expansion velocities of the $\mathrm{CO}$ outflows of most pPNe are below $70 \mathrm{~km} \mathrm{~s}^{-1}$, with a mean value around $40 \mathrm{~km} \mathrm{~s}^{-1}$. Curiously, some of the objects with the fastest, most massive outflows are the best studied ones and often inadvertently, and possibly mistakenly, taken as "representative" of the whole class by the community.

The spatio-kinematic structure of the equatorial waists of pPNe remains, in general, poorly characterized, since these regions are at best partially resolved in most cases. For this reason, the origin and formation mechanism of this component is unclear. With typical outer radii and expansion velocities of $\sim 1000-3000 \mathrm{AU}$ and $V_{\exp } \lesssim 30 \mathrm{~km} \mathrm{~s}^{-1}$, respectively, these dense torus-like components accommodate most of the nebular mass, and based on moderate angular resolution studies, they may be slightly older than the fast bipolar outflows [28]. The pPNe M 1-92 is one of the very few objects where the $\mathrm{CO}$ emission from the equatorial waist is spatially resolved [21]. These observations showed that, unexpectedly, the expansion velocity along the equatorial waist (thin and flat in this case) was a linear function of the radius as in the lobes, suggesting that both the slow waist and the fast lobes were shaped simultaneously - maybe by a single sudden (explosive?) acceleration event [21].

Angular resolution is clearly imperative for an accurate study of the different nebular components present in $\mathrm{pPNe}$, and thus, to make progress in our understanding of pPNe formation and evolution. In recent times, sub-arcsecond resolution observations have started to unveil the true nebular complexity underlying the simple hourglass (lobes + waist) structure that seems to be common to many pPNe. This is the case of, for example, HD101584, recently mapped with ALMA in CO emission with $\sim 25$ mas-resolution by Olofsson et al. $[29,30]$. These authors found that the real structure behind the simple, expanding, shell-like nebula inferred from previous low-angular resolution data [31] includes a series of nested sub-structures with complicated shapes and kinematics and different orientations (see a reconstruction of the 3-dimensional nebular structure in Fig.B.1 of Olofsson et al. [30]). The spectacular circumstellar characteristics derived from the ALMA maps have an impact on the scenario for the evolution of this object. In particular, Olofsson et al. [30] suggest that at the root of the formation of this complex array of structures there is a binary system in a post-common envelope evolution phase that ended before a stellar merger (although the details of the process or processes that led to the different components are uncertain).

Another example of nebular complexity disclosed by ALMA is found in the work by Sánchez Contreras et al. [32] on $\mathrm{OH} 231.8+4.2$, a remarkably fast bipolar outflow around a binary system formed by an AGB star and a main-sequence companion. As seen with $\sim 1^{\prime \prime}$-resolution, the molecular outflow, which is rather massive $\left(\sim 0.3 M_{\odot}\right)$ and fast $\left(V_{\exp }\right.$ up to $\left.\sim 400 \mathrm{~km} \mathrm{~s}^{-1}\right)$, has an overall cylindrical shape. At the center, there is a massive torus/waist expanding slowly $\left(V_{\exp } \sim 30 \mathrm{~km} \mathrm{~s}^{-1}\right)$ as in most standard pPNe [33]. As seen with ALMA at $\sim 0$ !'2-0!'3-resolution, the nebular structure is significantly more complex, and several distint components that were previously unknown were identified [32]. The large and dense equatorial waist of $\mathrm{OH} 231.8+4.2$ is now spatially resolved, which enables a proper characterization of its shape, and most importantly, its kinematics. The expansion velocity along the waist is not constant but increases from $3 \mathrm{~km} \mathrm{~s}^{-1}$ at its inner radius (at $\sim 200 \mathrm{AU}$ ) to $25 \mathrm{~km} \mathrm{~s}^{-1}$ at the outer edge (at $\sim 3000 \mathrm{AU}$ ). This velocity gradient suggests that the waist is not simply the remnant of the AGB shell pierced by jets and that it has been shaped almost simultaneously with the fast, large-scale bipolar lobes $\sim 800 \mathrm{yr}$ ago. Another success from these ALMA observations is the discovery of a compact and young ( $~ 50-80 \mathrm{yr}$ old) bipolar outflow selectively traced by the SiO molecule that is emerging from the stellar vicinity, indicating that there is an "active" jet engine at the heart of the nebula. However, whatever mechanism is at work at present, it is producing a bipolar outflow that is 
slower $\left(V_{\exp } \sim 23 \mathrm{~km} \mathrm{~s}^{-1}\right)$ and more symmetric than the large-scale lobes formed about $\sim 800 \mathrm{yr}$ ago. Surrounding the compact $\mathrm{SiO}$ outflow, there is a mini-hourglass composed of two mini-lobes and a mini-waist. As in M 1-92 (described earlier in this section), both these components show clear $V_{\exp } \propto r$ kinematics, consistent again with simultaneous formations of axial and equatorial structures.

One can readily extract some conclusions based on the ALMA observations of HD 101585 and $\mathrm{OH} 231.8+4.2$ described here. First is that the formation and evolution history of pPNe can be significantly more complex than inferred from CO-based studies with moderate angular resolution, and second is that the jet-launching mechanism or the conditions under which the jets formed in these environments can change over time. It is also clear from the cases exposed that equatorial regions may have complex kinematics that deserve further studies, since these regions, which seem to form simultaneously with the bipolar lobes (at least in sources accurately mapped), may hold important clues to understand the general PNe-shaping mechanisms.

\section{Disk-Prominent Post-AGB Stars: Rotating Circumbinary Disks}

Rotating disks are invoked by most wind collimation theories (see, e.g., [1] and references therein) and are suspected to be present in a particular class of post-AGB stars with near-IR emission excess (see [3] for a review). The IR emission excess points to hot dust located in a stable, disk-like structure close to the star $\left(a t \approx 10^{14}-10^{15} \mathrm{~cm}\right)$. In contrast to standard $\mathrm{pPNe}$, these objects lack massive, fast outflows; rather, they show narrow $\mathrm{CO}$ emission profiles consistent with rotating disks [34]. These objects are referred to by some authors as disk-prominent post-AGB objects.

The Red Rectangle is the prototype of this class of post-AGB stars and indeed is the first one where a disk in Keplerian rotation was spatially and spectrally resolved with IRAM-NOEMA (formerly known as Plateau de Bure Interferometer) by Bujarrabal et al. [35]. The disk of the Red Rectangle, which has been accurately modeled by these authors, has large dimensions, with an outer radius of about $2000 \mathrm{AU}$, and surrounds the central binary system, for which a central mass of about $1.5 \mathrm{M} \odot$ was deduced.

The disk of the Red Rectangle has been more recently mapped with ALMA with subarcsecresolution, resulting in an improvement of the disk model but also in the discovery of an X-shaped wind emerging from it $[36,37]$. This disk-wind is tenuous and slow $\left(V_{\exp } \sim 3-10 \mathrm{~km} \mathrm{~s}^{-1}\right)$, and therefore, very different from the massive, fast outflows of standard $\mathrm{pPNe}$. The mass in the wind is a factor $\sim 10$ lower than the mass of the rotating disk and it is believed to be material photoevaporating from the disk surface.

To date, inner rotating circumbinary disks have been mapped in CO emission in $\sim 5$ post-AGB objects. All of them have very similar properties, which are summarized in Table 1 of Sánchez Contreras et al. [38], including references to the original works. We note, however, that a very low-number statistics still prevail, and partially for this reason, it is not yet understood how these large-scale circumbinary disks form and what their role is in the PN-shaping process. Another open question that needs further investigation is: what is at the root of the dichotomy between disk-prominent post-AGBs and standard pPNe? Do these two major classes of post-AGB objects represent the end-stages of stars with different intrinsic properties? Or do these classes mainly develop as a result of different extrinsic factors? For example, the conditions under which their binaries interact during the AGB and/or post-AGB phases.

\subsection{Circumstellar Chemistry}

It is well known that AGB stars are very efficient molecular factories (see e.g., [39,40] and references therein). To date, about 100 molecular species have been discovered in the circumstellar envelopes around AGB stars by means of targeted line searches or full spectral line surveys mainly in the (sub)mm-wavelength range. As AGB stars evolve into the PN phase, and before the bulk of the envelope is fully ionized, important changes in the chemical contents of these objects happen as a result of the progressive dilution of the envelope, and consequently, the easier penetration of interstellar 
UV photons. These UV photons break down parent molecules, leading to molecular ions and radicals that trigger a series of chemical reactions that result in the formation of new molecular species. An active photo-induced chemistry is also expected in the inner layers of the envelope as the central star becomes hotter during the AGB-to-PN evolution. Finally, the shocks produced in the interaction between fast post-AGB winds and the slow AGB envelope previously ejected will initiate a series of shock-induced chemical reactions that will further transform the initial molecular content.

To date, most studies on circumstellar chemistry have focused on AGB stars, even though still, at present, full spectral surveys, covering instantaneously a wide range of frequencies and multiple transitions for many species, have been performed only for a few objects. Some examples are the C-rich AGB star IRC+10216 [41,42], probably the best studied AGB star, and the O-rich AGB stars IK Tau and $\mathrm{R}$ Dor $[43,44]$. The chemistries of a number of pPNe are also relatively well characterized, particularly the C-rich pPNe CRL 618 [45] and CRL 2688 [46] and the O-rich source OH 231.8+4.2 [47,48].

The chemistry of PNe is less well known, in general, than that of objects in earlier evolutionary stages. NGC 7027 and The helix are two of the best-characterized PNe [49,50]. Although the number of studies is growing (e.g., [51]), the global chemical content of evolved PNe has not been yet studied systematically for a sufficiently large sample of objects. This is in part because most molecules are thought to be destroyed in dispersing PNe due to intense stellar UV radiation field. This is true in many PNe (particularly the oldest), but certainly some PNe are molecule-rich, which may be linked to the presence of dusty tori and/or high-density clumps of gas and dust that shield molecules from the stellar radiation [52].

After $\mathrm{CO}$, the molecular species most frequently detected and most abundant in PNe are HCN, $\mathrm{HNC}$, and $\mathrm{HCO}^{+}$(see, e.g., Schmidt \& Ziurys [51], Bublitz et al. [53], for a more complete list). There have also been detections of many radicals, such as $\mathrm{CN}$ and $\mathrm{CH}$; polyatomic molecules such as $\mathrm{H}_{2} \mathrm{CO}$, $\mathrm{N}_{2} \mathrm{H}^{+}, \mathrm{C}_{3} \mathrm{H}_{2}$, etc.; and complex organic species, such as PAHs [54] and fullerenes [55]. Therefore, in contrast to initial expectations, PNe can be chemically rich and the survival of molecules in these environments is also key to understanding the chemistry of the interstellar medium.

Future work is needed, aiming at characterizing systematically and globally the chemistry in PNe using full spectral surveys. This should be accompanied by or combined with studies with high-angular resolution, which are needed for a full understanding of the chemical processes that govern molecular formation in different regions inside PNe. In addition to observational characterization efforts, improvements of chemical models are desired for an optimal scientific return from the observations-PNe are complex systems and for this reason models need to incorporate the effects of, e.g., shocks, $\mathrm{X}$-rays, and grain surface reactions for a proper modeling and understanding of these systems.

\section{Ionized Gas}

Although the (sub)mm-wavelength regime is commonly associated with molecular line studies of pPNe/yPNe (and other astrophysical environments), this wavelength domain also offers the possibility to observe the ionized gas component. The pioneering works by Bachiller et al. [56], Martín-Pintado et al. [57], showed that mm-wavelength radio recombination lines (mmRRLs) can be used to study the deepest regions at the heart of pPNe, within 100-200 AU from the center, where the gas has started being ionized by the central star.

Recently, Sánchez Contreras et al. [58] carried out a pilot survey of mmRRL emission in a small sample of pPNe with emerging $\mathrm{H}$ II regions, aimed at investigating the physical properties and kinematics of ionized regions close to the jet launching site and to study the present day mass-loss rate. Several lines were detected towards the objects CRL 618, M 2-9, and MWC 922; the analysis performed included radiative transfer modeling of the free-free continuum and mmRRLs that enabled a detailed characterization of their compact ionized central regions. In one of the objects, MWC 922 (also known as the Red Square Nebula), the mmRRL profiles indicated a disk+wind system in rotation at the core. 
In CRL 618 and M2-9, the data pointed to winds ejected just a few decades ago at relatively high mass-loss rates.

The mass-loss rate during the post-AGB phase is indeed a critical factor in the AGB-to-PN evolution that is, however, very poorly known. For this reason, evolutionary models are bound to adopt mass-loss prescriptions that are not empirically constrained by direct observations. Sánchez Contreras et al. [58] found that the mass-loss rates measured in their sample of eight pPNe-candidates are systematically and significantly above the values normally adopted by models (see Figure 1). This is an important result that needs to be confirmed by studying larger samples, since it will have implications in the AGB-to-PN crossing times, which could be significantly shorter than what is normally assumed.

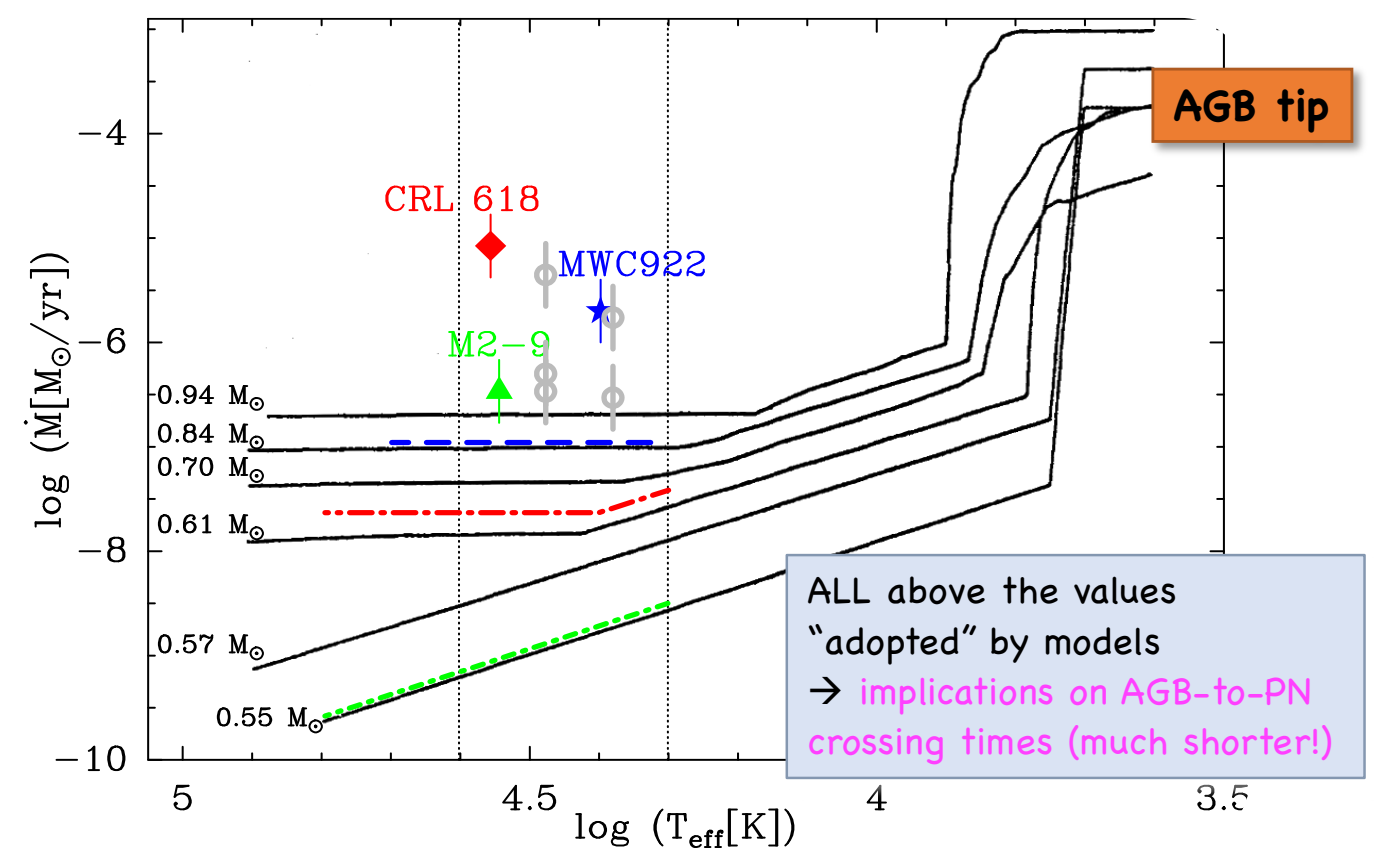

Figure 1. Adapted from Figure 8 of Sánchez Contreras et al. [58]. Post-asymptotic giant branch (post-AGB) mass-loss vs. effective temperatures adopted by post-AGB models (solid black lines) and empirically deduced (symbols). The thick dashed and dot-dashed lines indicate the mass-loss rates typically assumed by evolutionary models for the three objects in with mmRRL detections. See Sánchez Contreras et al. [58] for details.

The ionized core of the B[e]-type star MWC 922 has been spectrally and spatially resolved with ALMA [59]. The presence of a compact rotating disk at the center is confirmed. Moreover, and most importantly, a fast $\left(\sim 100 \mathrm{~km} \mathrm{~s}^{-1}\right)$ bipolar outflow orthogonal to the disk and rotating in a similar sense has been discovered. The continuum and mmRRLs maps show an X-shaped H II region, which is consistent with a biconical shell of ionized gas on the surface of a larger rotating disk that is illuminated and photoionized by the central star. The fast bipolar wind is found to be running inside the ionized layers of the rotating disk. The launching radius of the fast wind is smaller than the inner edge of the disk ( $\sim 30 \mathrm{AU})$. This indicates that the fast wind is not simply photoevaporating from the disk, but it is being actively launched from a more compact region inside the disk observed.

The ALMA maps have been modeled, which has enabled characterizing with unprecedented detail the ionized core of this object. One important parameter deduced from these data and the model is the mass of the system at the center of the rotating disk, which is $\sim 10 M_{\odot}$ or $\sim 18 M_{\odot}$ depending on the distance to the source adopted $(d=1.7$ or $3 \mathrm{kpc})$. This parameter is crucial to constrain the uncertain nature of this object, which may be a blue supergiant in a binary system as discussed by Sánchez Contreras et al. [59]. 
To date, mmRRLs have been barely observationally explored (partially due to the lack of sufficient sensitivity in the pre-ALMA era); however, the exciting results described above illustrate the great potential of mmRRLs to get closer to the jet launching site and to study current post-AGB mass ejections in objects that are quickly transitioning into the PN stage.

\section{Final Remarks}

From numerous and vivid discussions during this meeting, it is clear that many are the questions that remain to be answered concerning AGB-to-PN evolution in general, but particularly concerning $\mathrm{PN}$-shaping. The(sub)mm-wavelength range offers a wide variety of line and continuum diagnostics, some of them described in this contribution, which can be used to improve our understanding of these complex systems. Our efforts should not focus only on the characterizations of the properties of fast bipolar outflows of pPNe, but also on their equatorial regions (including post-AGB stars with large-scale expanding tori and circumbinary rotating disks), which may hold important clues to reconstruct the $\mathrm{PN}$-shaping history, and on the relation between standard pPNe and disk-prominent post-AGB objects. To make progress in the field, we need both statistical studies of large unbiased samples of $\mathrm{pPNe} / \mathrm{yPNe}$ and detailed observations with high angular resolution of individual targets, which are crucial to disclosing the real nebular complexities of these objects and to penetrate closer to their jet-launching sites.

Funding: This work has been partially supported by the Spanish MINECO through grants AYA2016-75066-C2-1-P and AYA2016-78994-P and by the European Research Council through ERC grant 610256: NANOCOSMOS.

Conflicts of Interest: The author declares no conflict of interest. Founding sponsors had no role in the design of the study; in the collection, analyses, or interpretation of data; in the writing of the manuscript, and in the decision to publish the results.

\section{References}

1. Balick, B.; Frank, A. Shapes and Shaping of Planetary Nebulae. Annu. Rev. Astron. Astrophys. 2002, 40, 439. [CrossRef]

2. Kwok, S. Proto-planetary nebulae. Annu. Rev. Astron. Astrophys. 1993, 31, 63. [CrossRef]

3. van Winckel, H. Post-AGB Stars. Annu. Rev. Astron. Astrophys. 2003, 41, 391. [CrossRef]

4. Bujarrabal, V. Molecular line emission from planetary and protoplanetary nebulae. In Planetary Nebulae in Our Galaxy and Beyond, Proceedings of the International Astronomical Union, Symposium \#234; Barlow, M.J., Méndez, R.H., Eds.; Cambridge University Press: Cambridge, UK, 2006; pp. 193-202.

5. Bachiller, R.; Gomez-Gonzalez, J.; Bujarrabal, V.; Martin-Pintado, J. Carbon monoxide in proto-planetary nebulae. Astron. Astrophys. 1988, 196, L5.

6. Bujarrabal, V.; Alcolea, J.; Planesas, P. The molecular emission of young preplanetary nebulae. Astron. Astrophys. 1992, 257, 701.

7. Cernicharo, J.; Guelin, M.; Martin-Pintado, J.; Penalver, J.; Mauersberger, R. A 200 KM s-1 molecular outflow in the protoplanetary nebula CRL 618. Astron. Astrophys. 1989, 222, L1.

8. Knapp, G.R.; Sutin, B.M.; Phillips, T.G.; Ellison, B.N.; Keene, J.B.; Leighton, R.B.; Masson, C.R.; Steiger, W.; Veidt, B.; Young, K. CO Emission from Evolved Stars and Proto-Planetary Nebulae. Astrophys. J. 1989, 336, 822. [CrossRef]

9. Likkel, L.; Forveille, T.; Omont, A.; Morris, M. CCO observations of cold IRAS objects: AGB and post-AGB stars. Astron. Astrophys. 1991, 246, 153.

10. Volk, K.; Kwok, S.; Woodsworth, A.W. CO Observations of Candidates for Carbon-rich Asymptotic Giant Branch and Post-Asymptotic Giant Branch Stars. Astrophys. J. 1993, 402, 292. [CrossRef]

11. Huggins, P.J.; Bachiller, R.; Planesas, P.; Forveille, T.; Cox, P. A CO Survey of Young Planetary Nebulae. Astrophysical J. Suppl. Ser. 2005, 160, 272. [CrossRef]

12. Bujarrabal, V.; Castro-Carrizo, A.; Alcolea, J.; Sánchez Contreras, C. Mass, linear momentum and kinetic energy of bipolar flows in protoplanetary nebulae. Astron. Astrophys. 2001, 377, 868. [CrossRef] 
13. Castro-Carrizo, A.; Quintana-Lacaci, G.; Neri, R.; Bujarrabal, V.; Schoier, F. L.; Winters, J.M.; Olofsson, H.; Lindqvist, M.; Alcolea, J.; Lucas, R.; et al. Mapping the ${ }^{12} \mathrm{CO} J=1-0$ and J=2-1 emission in AGB and early post-AGB circumstellar envelopes. I. The COSAS program, first sample. Annu. Rev. Astron. Astrophys. 2010, 523, A59. [CrossRef]

14. Cox, P.; Lucas, R.; Huggins, P. J.; Forveille, T.; Bachiller, R.; Guilloteau, S.; Maillard, J.P.; Omont, A. Multiple molecular outflows in AFGL 2688. Annu. Rev. Astron. Astrophys. 2000, 353, L25.

15. Fong, D.; Meixner, M.; Sutton, E. C.; Zalucha, A.; Welch, W.J. Evolution of the Circumstellar Molecular Envelope. I. A BIMA CO Survey of Evolved Stars. Astrophys. J. 2006, 652, 1626. [CrossRef]

16. Huggins, P.J.; Muthu, C.; Bachiller, R.; Forveille, T.; Cox, P. High resolution millimeter imaging of the proto-planetary nebula He 3-1475. Annu. Rev. Astron. Astrophys. 2004, 414, 581. [CrossRef]

17. Neri, R.; Kahane, C.; Lucas, R.; Bujarrabal, V.; Loup, C. A (12) CO (J=1->0) and (J=2-> 1) atlas of circumstellar envelopes of AGB and post-AGB stars. Astron. Astrophys. Suppl. Ser. 1998, 130, 1-64. [CrossRef]

18. Sánchez Contreras, C.; Bujarrabal, V.; Castro-Carrizo, A.; Alcolea, J.; Sargent, A. Interferometric CO J = 2-1 Emission Mapping of the Protoplanetary Nebula IRAS 19475+3119. Astrophys. J. 2006, 643, 945. [CrossRef]

19. Sánchez Contreras, C.; Sahai, R. OPACOS: OVRO Post-AGB CO (1-0) Emission Survey. I. Data and Derived Nebular Parameters. Astron. Astrophys. Suppl. Ser. 2012, 203, 16. [CrossRef]

20. Tafoya, D.; Orosz, G.; Vlemmings, W.H.T.; Sahai, R.; Pérez-Sánchez, A.F. Spatio-kinematical model of the collimated molecular outflow in the water-fountain nebula IRAS 16342-3814. Annu. Rev. Astron. Astrophys. 2019, 629, A8. [CrossRef]

21. Alcolea, J.; Neri, R.; Bujarrabal, V. Minkowski's footprint revisited. Planetary nebula formation from a single sudden event? Annu. Rev. Astron. Astrophys. 2007, 468, L41. [CrossRef]

22. Castro-Carrizo, A.; Bujarrabal, V.; Sánchez Contreras, C.; Alcolea, J.; Neri, R. The structure and dynamics of the molecular envelope of M 2-56. Annu. Rev. Astron. Astrophys. 2002, 386, 633. [CrossRef]

23. Gómez, J.F.; Niccolini, G.; Suárez, O.; Miranda, L.F.; Rizzo, J.R.; Uscanga, L.; Green, J.A.; de Gregorio-Monsalvo, I. ALMA imaging of the nascent planetary nebula IRAS 15103-5754. Mon. Not. R. Astron. Soc. 2018, 480, 4991. [CrossRef]

24. Sahai, R.; Vlemmings, W.H.T.; Huggins, P. J.; Nyman, L.-A.; Gonidakis, I. ALMA Observations of the Coldest Place in the Universe: The Boomerang Nebula. Astrophys. J. 2013, 777, 92. [CrossRef]

25. Castro-Carrizo, A.; Bujarrabal, V.; Neri, R.; Alcolea, J.; Sánchez Contreras, C.; Santander-Garcia, M.; Nyman, L.-A. Structure and dynamics of the molecular gas in M 2-9: A follow-up study with ALMA. Annu. Rev. Astron. Astrophys. 2017, 600, A4. [CrossRef]

26. Sánchez Contreras, C.; Bujarrabal, V.; Castro-Carrizo, A.; Alcolea, J.; Sargent, A. 1-arcsec Resolution Mapping of the Molecular Envelope of the Protoplanetary Nebula CRL 618. Astrophys. J. 2004, 617, 1142. [CrossRef]

27. Sánchez Contreras, C.; Martin, S.; Sahai, R. The fast, massive outflow of the pre-planetary nebula IRAS 19374+2356. In Highlights of Spanish Astrophysics VII, Proceedings of the X Scientific Meeting of the Spanish Astronomical Society (SEA), Valencia, CA, USA, 9-13 July 2012; Guirado, J.C., Lara, L.M., Quilis, V., Gorgas, J., Eds.; Sociedad Española de Astronomía: Barcelona, Spain, 2012; p. 671.

28. Huggins, P. J. Jets and Tori in Proto-Planetary Nebulae. Astrophys. J. 2007, 663, 342. [CrossRef]

29. Olofsson, H.; Vlemmings, W.H.T.; Maercker, M.; Humphreys, E.M.L.; Lindqvist, M.; Nyman, L.; Ramstedt, S. ALMA view of the circumstellar environment of the post-common-envelope-evolution binary system HD 101584. Annu. Rev. Astron. Astrophys. 2015, 576, L15. [CrossRef]

30. Olofsson, H.; Khouri, T.; Maercker, M.;Bergman, P.; Doan, L.; Tafoya, D.; Vlemmings, W.H.T.; Humphreys, E.M.L.; Lindqvist, M.; Nyman, L.; et al. HD 101584: Circumstellar characteristics and evolutionary status. Annu. Rev. Astron. Astrophys. 2019, 623, A153. [CrossRef]

31. Olofsson, H.; Nyman, L.-Å. The circumstellar molecular envelope of HD 101584. Annu. Rev. Astron. Astrophys. 1999, 347, 194.

32. Sánchez Contreras, C.; Alcolea, J.; Bujarrabal, V.; Castro-Carrizo, A.; Velilla Prieto, L.; Santander-Garcia, M.; Quintana-Lacaci, G.; Cernicharo, J. Through the magnifying glass: ALMA acute viewing of the intricate nebular architecture of $\mathrm{OH}$ 231.8+4.2. Annu. Rev. Astron. Astrophys. 2018, 618, A164. [CrossRef]

33. Alcolea, J.; Bujarrabal, V.; Sánchez Contreras, C.; Neri, R.; Zweigle, J. The highly collimated bipolar outflow of $\mathrm{OH}$ 231.8+4.2. Annu. Rev. Astron. Astrophys. 2001, 373, 932. [CrossRef]

34. Bujarrabal, V.; Alcolea, J.; Van Winckel, H.; Santander-Garcia, M.; Castro-Carrizo, A. Extended rotating disks around post-AGB stars. Astron. Astrophys. 2013, 557, A104. [CrossRef] 
35. Bujarrabal, V.; Neri, R.; Alcolea, J.; Kahane, C. Detection of an orbiting gas disk in the Red Rectangle. Astron. Astrophys. 2003, 409, 573. [CrossRef]

36. Bujarrabal, V.; Castro-Carrizo, A.; Alcolea, J.; Van Winckel, H.; Sánchez Contreras, C.; Santander-Garcia, M.; Neri, R.; Lucas, R. ALMA observations of the Red Rectangle, a preliminary analysis. Astron. Astrophys. 2013, 557, L11. [CrossRef]

37. Bujarrabal, V., Castro-Carrizo, A.; Alcolea, J.; Santander-García, M.; van Winckel, H.; Sánchez Contreras, C. Further ALMA observations and detailed modeling of the Red Rectangle. Astron. Astrophys. 2016, 593, A92. [CrossRef]

38. Sánchez Contreras, C.; Alcolea, J.; Bujarrabal, V.; Castro-Carrizo, A.; Velilla Prieto, L.; Santander-Garcia, M.; Quintana-Lacaci, G.; Cernicharo, J. ALMA's Acute View of pPNe: Through the Magnifying Glass... and What We Found There. Galaxies 2018, 6, 94. [CrossRef]

39. Höfner, S.; Olofsson, H. Mass loss of stars on the asymptotic giant branch. Mechanisms, models and measurements. Astron. Astrophys. Rev. 2018, 26, 1. [CrossRef]

40. Millar, T.J. Chemistry in the circumstellar medium. Unveiling the dust formation zone. Astrophys. Space Sci. 2008, 313, 223. [CrossRef]

41. Agúndez, M.; Cernicharo, J. Oxygen Chemistry in the Circumstellar Envelope of the Carbon-Rich Star IRC +10216. Astrophys. J. 2006, 650, 374. [CrossRef]

42. Cernicharo, J.; Guélin, M.; Kahane, C. A lambda $2 \mathrm{~mm}$ molecular line survey of the C-star envelope IRC+10216. Astron. Astrophys. Suppl. Ser. 2000, 142, 181-215. [CrossRef]

43. De Beck, E.; Olofsson, H. Circumstellar environment of the M-type AGB star R Doradus. APEX spectral scan at 159.0-368.5 GHz. Annu. Rev. Astron. Astrophys. 2018, 615, A8. [CrossRef]

44. Velilla Prieto, L.; Sánchez Contreras, C.; Cernicharo, J.; Agúndez, M.; Quintana-Lacaci, G.; Bujarrabal, V.; Alcolea, J.; Balança, C.; Herpin, F.; Menten, K.M.; et al. The millimeter IRAM-30 m line survey toward IK Tauri. Annu. Rev. Astron. Astrophys. 2017, 597, A25. [CrossRef] [PubMed]

45. Pardo, J.R.; Cernicharo, J. Molecular Abundances in CRL 618. Astrophys. J. 2007, 654, 978. [CrossRef]

46. Zhang, Y.; Sun, K.; Nakashima, J.-I.; Chau, W.; Dinh-V-Trung. A Molecular Line Survey of the Carbon-rich Protoplanetary Nebula AFGL 2688 in the $3 \mathrm{~mm}$ and $1.3 \mathrm{~mm}$ Windows. Astrophys. J. 2013, 773, 71. [CrossRef]

47. Morris, M.; Guilloteau, S.; Lucas, R.; Omont, A. The Rich Molecular Spectrum and the Rapid Outflow of OH 231.8+4.2. Astrophys. J. 1987, 321, 888. [CrossRef]

48. Velilla Prieto, L.; Sánchez Contreras, C.; Cernicharo, J.; Agúndez, M.; Quintana-Lacaci, G.; Alcolea, J.; Bujarrabal, V.; Herpin, F.; Menten, K.M.; Wyrowski, F. New N-bearing species towards OH 231.8+4.2. HNCO, HNCS, $\mathrm{HC}_{3} \mathrm{~N}$, and NO. Annu. Rev. Astron. Astrophys. 2015, 575, A84. [CrossRef]

49. Bachiller, R.; Forveille, T.; Huggins, P.J.; Cox, P. The chemical evolution of planetary nebulae. Annu. Rev. Astron. Astrophys. 1997, 324, 1123.

50. Tenenbaum, E.D.; Milam, S.N.; Woolf, N.J.; Ziurys, L.M. Molecular Survival in Evolved Planetary Nebulae: Detection of $\mathrm{H}_{2} \mathrm{CO}, \mathrm{c}_{-} \mathrm{C}_{3} \mathrm{H}_{2}$, and $\mathrm{C}_{2} \mathrm{H}$ in the Helix. Astrophys. J. Lett. 2009, 704, L108. [CrossRef]

51. Schmidt, D.R.; Ziurys, L.M. New Detections of HNC in Planetary Nebulae: Evolution of the $[\mathrm{HCN}] /[\mathrm{HNC}]$ Ratio. Astrophys. J. 2017, 835, 79. [CrossRef]

52. Woods, P.M.; Nyman, L.-Å.; Schöier, F.L.; Zijlstra, A.A.; Millar, T.J.; Olofsson, H. Molecules in bipolar proto-planetary nebulae. Annu. Rev. Astron. Astrophys. 2005, 429, 977. [CrossRef]

53. Bublitz, J.; Kastner, J. H.; Santander-García, M.; Bujarrabal, V.; Alcolea, J.; Montez, R. A new radio molecular line survey of planetary nebulae. $\mathrm{HNC} / \mathrm{HCN}$ as a diagnostic of ultraviolet irradiation. Annu. Rev. Astron. Astrophys. 2019, 625, A101. [CrossRef]

54. Bernard Salas, J.; Pottasch, S.R.; Beintema, D.A.; Wesselius, P.R. The ISO-SWS spectrum of planetary nebula NGC 7027. Annu. Rev. Astron. Astrophys. 2001, 367, 949. [CrossRef]

55. Cami, J.; Bernard-Salas, J.; Peeters, E.; Malek, S.E. Detection of C60 and C70 in a Young Planetary Nebula. Science 2010, 329, 1180. [CrossRef]

56. Bachiller, R.; Huggins, P.J.; Martin-Pintado, J.; Cox, P. Millimeter recombination lines in the planetary nebula BD+30³639. Astron. Astrophys. 1992, 256, 231.

57. Martin-Pintado, J.; Bujarrabal, V.; Bachiller, R.; Gomez-Gonzalez, J.; Planesas, P. Radiocontinuum and recombination lines toward CRL 618. Evidence for an ionized stellar wind? Astron. Astrophys. 1988, 197, L15. 
58. Sánchez Contreras, C.; Báez-Rubio, A.; Alcolea, J.; Bujarrabal, V.; Martín-Pintado, J. A pilot search for $\mathrm{mm}$-wavelength recombination lines from emerging ionized winds in pre-planetary nebulae candidates. Astron. Astrophys. 2017, 603, A67. [CrossRef]

59. Sánchez Contreras, C.; Báez-Rubio, A.; Alcolea, J.; Castro-Carrizo, A.; Bujarrabal, V.; Martín-Pintado, J.; Tafoya, D. A rotating fast bipolar wind and disk system around the B[e]-type star MWC 922. Astron. Astrophys. 2019, 629, A136. [CrossRef]

(C) 2020 by the author. Licensee MDPI, Basel, Switzerland. This article is an open access article distributed under the terms and conditions of the Creative Commons Attribution (CC BY) license (http:/ / creativecommons.org/licenses/by/4.0/). 